\title{
O GÊNERO TRACHELOMONAS (EUGLENOPHYTA) DOS MUNICÍPIOS DE SÃO SEBASTIÃO DO PASSÉ, CANDEIAS, MATA DE SÃO JOÃO, POJUCA E SÃO FRANCISCO DO CONDE, REGIÃO METROPOLITANA DE SALVADOR, BAHIA, BRASIL.
}

\author{
$\underline{\text { Adones de Jesus Santos Pereira }}{ }^{1}$; Carlos Wallace do Nascimento Moura ${ }^{2}$ \\ 1. Bolsista PIBIC/FAPESB, Graduando em Bacharelado em Ciências Biológicas, Universidade Estadual de Feira de \\ Santana, e-mail: adones2006@gmail.com \\ 2. Orientador, Departamento de Ciências Biológicas, Universidade Estadual de Feira de Santana, e-mail: \\ wallace@uefs.br
}

PALAVRAS-CHAVE: Microalga; Taxonomia; Inventário.

\section{INTRODUÇÃO}

Euglenophyta é uma das muitas divisões de microalgas existentes, englobando atualmente 40 gêneros e aproximadamente 800 espécies (Parra \& Bicudo, 1995), embora segundo Norton et al. (1996) há estimativa de 2 mil espécies pertencentes à divisão.

Trachelomonas Ehrenberg emend. Deflandre é o gênero mais representativo da divisão Euglenophyta, com 377 táxons aceitos atualmente (Guiry \& Guiry 2016). A maioria dos táxons são referidos para ambientes de água doce (Bourrelly, 1970), embora também existam espécies marinhas (Leedale, 1967).

O gênero inclui algas de vida livre e hábito solitário, caracterizadas por possuir célula com um envelope rígido e mucilaginoso denominado de lórica, apresentando um poro no polo anterior do qual emerge o flagelo. A lórica apresenta diferentes formatos e padrões de ornamentação, os quais são empregados na taxonomia do grupo (Tell \& Conforti, 1986), e uma coloração castanho-amarelada, ocasionada pela impregnação de compostos férricos e de manganês (Leedale, 1975; Dunlap et al. 1983).

Trabalhos taxonômicos de microalgas no estado do Bahia ainda são escassos, diante da complexidade de suas bacias de drenagem e da diversidade de ecossistemas aquáticos continentais. Com relação ao gênero Trachelomonas, todas as informações disponíveis estão restritas aos seguintes trabalhos: Fuentes et al. 2010; Santana, 2011; Severiano et al. 2012 e Alves-da-Silva \& Menezes, 2015, embora os autores mencionados não forneçam descrição e ilustrações em seus trabalhos que auxiliassem a confirmação dos táxons, e a um estudo taxonômico (Pereira, 2016), nos quais foram referidos 45 táxons.

Diante do exposto, o presente trabalho teve como objetivo a realização de um estudo taxonômico das microalgas pertencentes ao gênero Trachelomonas ocorrentes em corpos d'água dos municípios de São Sebastião do Passé, Candeias, Mata de São João, Pojuca e São Francisco do Conde, Região Metropolitana de Salvador, Bahia, Brasil, de modo a ampliar o registro de espécies para a Bahia e, consequentemente, para o Brasil.

\section{MATERIAL E MÉTODOS}

O material foi previamente obtido através de coletas realizadas ao longo dos corpos d'água situados na Região Metropolitana de Salvador (RMS), nos municípios de São Sebastião do Passé, Candeias, Mata de São João, Pojuca e São Francisco do Conde, durante o período de maio de 2014 a outubro de 2015. As coletas foram feitas ao acaso, em corpos aquáticos (rios, lagoas, represas, etc.) pouco antropizados, oligotróficos e com presença de macrófitas total ou parcialmente submersas. Todos os pontos de coleta foram georeferenciados. 
A coleta do material foi feita de acordo com os métodos usuais empregados em estudos de taxonomia de microalgas continentais (Bicudo \& Menezes, 2006).

Ao todo foram analisadas 60 amostras, sendo 18 do município de São Sebastião do Passé, cinco de Candeias, 22 de Mata de São João, oito de Pojuca e sete de São Francisco do Conde.

As amostras foram analisadas com auxílio do microscópio óptico binocular marca Olympus (modelo BX43) e as fotomicrografias obtidas com máquina fotográfica digital (Modelo QImaging 5.0) acoplado ao microscópio.

As dimensões dos espécimes foram aferidas através de ocular micrométrica e estão representadas pelos limites métricos mínimos e máximos. Aproximadamente 290 alíquotas foram preparadas das unidades amostrais e analisadas. A identificação taxonômica foi, sempre que possível, em nível infragenérico, analisando-se as características morfológicas e métricas das populações. Estas foram baseadas em trabalhos especializados, incluindo floras, revisões, dissertações e teses.

O sistema de classificação utilizado segue Guiry \& Guiry (2016). O material analisado encontra-se tombado na coleção líquida do Herbário da Universidade Estadual de Feira de Santana (HUEFS).

\section{RESULTADOS E DISCUSSÃO}

Das 60 unidades amostrais analisadas, 49 apresentaram representantes de Trachelomonas, sendo 17 unidades em São Sebastião do Passé, cinco em Candeias, 14 em Mata de São João, cinco em Pojuca e sete em São Francisco do Conde.

O gênero Trachelomonas foi representado na flora local por 31 táxons, distribuídos em 19 espécies, nove variedades e uma forma taxonômica não típica, além de duas variedades não identificadas pertencentes a $T$. kellogii e um táxon identificado a apenas a nível de gênero (Trachelomonas sp. 1).

O município de São Sebastião do Passé apresentou a maior riqueza de táxons, com 25 táxons, seguido por Mata de São João, São Francisco do Conde e Pojuca com 21, 19 e 17 táxons, respectivamente. Candeias apresentou a menor riqueza (11 táxons).

Dentre os 31 táxons identificados, oito foram comuns aos cinco municípios ( $T$. armata var. longispina, T. armata var. litoralensis, T. conica, T. hispida var. duplex, T. intermedia var. minor, T. kellogii var. kellogii, T. robusta var. robusta, T. volvocinopsis var. volvocinopsis). Por outro lado, constatou-se que alguns táxons foram exclusivos de determinados municípios, sendo quatro exclusivos de São Sebastião do Passé (T. allia var. obesa, T. lemmermannii var. lemmermannii, T. megalacantha var. megalacantha, Trachelomonas sp. 1), um de Mata de São João (T. magdaleniana), um de Pojuca ( $T$. superba) e um de São Francisco do Conde (T. spinosa) (Tab. 1).

Trachelomonas volvocinopsis var. volvocinopsis foi o táxon mais representativo, considerando os cinco municípios amostrados, tendo sido registrado em 31 unidades amostrais. 
Tabela. 1. Listagem dos táxons de Trachelomonas (Euglenophyceae) anteriormente referidas para o Estado e dos dados obtidos nos municípios de São Sebastião do Passé, Candeias, Mata de São João, Pojuca e São Francisco do Conde, Região Metropolitana de Salvador, Bahia, Brasil; Citações anteriores: 1- Fuentes et al. (2010), 2- Santana (2011), 3- Severiano et al. (2012), 4- Alves-da-Silva \& Menezes (2015), 5- Pereira (2016); Presente trabalho: SSP= São Sebastião do Passé, CA= Candeias, MSJ= Mata de São João, $\mathrm{PO}=$ Pojuca, $\mathrm{SFC}=$ São Francisco doConde.

\begin{tabular}{|c|c|c|c|c|c|c|}
\hline \multirow[b]{2}{*}{ Táxons } & \multirow{2}{*}{$\begin{array}{c}\text { Citações } \\
\text { anteriores }\end{array}$} & \multicolumn{5}{|c|}{ Presente trabalho } \\
\hline & & SSP & CA & MSJ & PO & SFC \\
\hline T. abrupta Swirenko emend. Deflandre var. abrupta & $2 ; 5$ & $\mathrm{x}$ & $\mathrm{X}$ & & $\mathrm{x}$ & $\mathrm{x}$ \\
\hline T. abrupta Swirenko emend. Deflandre var. arcuata (Playfair) Deflandre & $2 ; 5$ & & & & & \\
\hline T. acanthophora Stokes & 2 & $\mathrm{x}$ & & & & $\mathrm{x}$ \\
\hline T. acanthophora A.C.Stokes var. speciosa (Deflandre) Balech & & $\mathrm{x}$ & & & & $\mathrm{x}$ \\
\hline T. allia Drezepolski emend. Deflandre var. obesa Balech & 5 & $\mathrm{x}$ & & & & \\
\hline T. armata (Ehrenberg) F.Stein var. armata f. armata & $2 ; 3 ; 5$ & $\mathrm{x}$ & $\mathrm{X}$ & & & $\mathrm{X}$ \\
\hline T. armata (Ehrenberg) F.Stein var. armata f. inevoluta Deflandre & 5 & $\mathrm{x}$ & & $\mathrm{X}$ & $\mathrm{X}$ & $\mathrm{X}$ \\
\hline T. armata (Ehrenberg) F.Stein var. litoralensis Tell \& Domitrovic & $2 ; 5$ & $\mathrm{x}$ & $\mathrm{x}$ & $\mathrm{x}$ & $\mathrm{x}$ & $\mathrm{X}$ \\
\hline T. armata (Ehrenberg) F.Stein var. longispina Playfair emend. Deflandre & $2 ; 5$ & $\mathrm{x}$ & $\mathrm{x}$ & $\mathrm{x}$ & $\mathrm{x}$ & $\mathrm{x}$ \\
\hline T. armata var. ovata Svirenko & 2 & & & & & \\
\hline T. armata (Ehrenberg) F.Stein var. sparsigranosa Playfair & 5 & & & & & \\
\hline T. armata (Ehrenberg) F.Stein var. steinii Lemmermann emend. Deflandre & $2 ; 5$ & $\mathrm{x}$ & $\mathrm{x}$ & $\mathrm{x}$ & & $\mathrm{x}$ \\
\hline T. balechii Tell & 5 & & & $\mathrm{x}$ & & \\
\hline T. bulla F.Stein emend. Deflandre. var. bulla & 2 & & & & & \\
\hline T. conica Playfair & 5 & $\mathrm{x}$ & $\mathrm{x}$ & $\mathrm{x}$ & $\mathrm{x}$ & $\mathrm{x}$ \\
\hline T. curta A.M.Cunha emend. Deflandre var. curta & 5 & & & & & \\
\hline T. curta A.M.Cunha emend. Deflandre var. subpunctata Bourrelly & 5 & & & & & \\
\hline T. dangeardiana (Deflandre) Huber-Pestalozzi var. glabra (Playfair) Deflandre & 5 & & & & & \\
\hline T. dastuguei Balech var. dastuguei f. dastuguei & 2 & & & & & \\
\hline T. hemispherica Garcia-de-Emiliani & 2 & & & & & \\
\hline T. hirta A.M.Cunha var. hirta & 2 & & & & & \\
\hline T. hispida (Perty) F.Stein emend. Deflandre var. hispida & $2 ; 5$ & $\mathrm{x}$ & & $\mathrm{x}$ & $\mathrm{X}$ & \\
\hline T. hispida (Perty) F.Stein emend. Deflandre var. coronata Lemmermann & 5 & $\mathrm{x}$ & & $\mathrm{x}$ & & \\
\hline T. hispida (Perty) F.Stein emend. Deflandre var. crenulatocollis (Maskell) & & $\mathrm{x}$ & & & & $\mathrm{X}$ \\
\hline T. hispida (Perty) F.Stein emend. Deflandre var. duplex Deflandre & 5 & $\mathrm{x}$ & $\mathrm{x}$ & $\mathrm{x}$ & $\mathrm{x}$ & $\mathrm{x}$ \\
\hline T. hispida (Perty) F.Stein emend. Deflandre var. punctata Lemmermann & 5 & & & & & \\
\hline T. intermedia P.A.Dangeard var. intermedia & 5 & $\mathrm{X}$ & & $\mathrm{x}$ & $\mathrm{X}$ & $\mathrm{x}$ \\
\hline T. intermedia P.A.Dangeard var. minor Tell & 5 & $\mathrm{x}$ & $\mathrm{x}$ & $\mathrm{x}$ & $\mathrm{x}$ & $\mathrm{x}$ \\
\hline T. kellogii Skvortzov emend. Deflandre var. kellogii & 5 & $\mathrm{x}$ & $\mathrm{x}$ & $\mathrm{x}$ & $\mathrm{x}$ & $\mathrm{X}$ \\
\hline T. kellogii var. 1 & & & & $\mathrm{x}$ & $\mathrm{x}$ & \\
\hline T. kellogii var. 2 & & $\mathrm{x}$ & & $\mathrm{x}$ & $\mathrm{x}$ & $\mathrm{x}$ \\
\hline T. lacustris Drezepolski var. lacustris & $2 ; 5$ & & & $\mathrm{x}$ & $\mathrm{x}$ & $\mathrm{X}$ \\
\hline T. lemmermannii Woloszýnska emend. Deflandre var. lemmermannii & 5 & $\mathrm{x}$ & & & & \\
\hline T. lemmermannii Woloszýnska emend. Deflandre var. acuminata Deflandre & 2 & $\mathrm{x}$ & & $\mathrm{x}$ & $\mathrm{x}$ & \\
\hline T. magdaleniana Deflandre & & & & $\mathrm{x}$ & & \\
\hline T. megalacantha A.M.Cunha var. megalacantha & 5 & $\mathrm{x}$ & & & & \\
\hline T. obesa Ehrenberg & $1 ; 3$ & & & & & \\
\hline T. oblonga Lemmermann var. oblonga & $1 ; 3 ; 5$ & & & & & \\
\hline T. parvicollis Deflandre & 2 & & & & & \\
\hline T. raciborskii Woloszýnska var. raciborskii & $2 ; 5$ & & & & & \\
\hline T. raciborskii Woloszýnska var. nova Drezepolski & 5 & $\mathrm{x}$ & & & & $\mathrm{x}$ \\
\hline T. raciborskii Woloszýnska var. nova Drezepolski f. minor T. Hortobágyi & 5 & & & & & \\
\hline T. robusta Svirenko var. robusta & 5 & $\mathrm{x}$ & $\mathrm{x}$ & $\mathrm{x}$ & $\mathrm{X}$ & $\mathrm{X}$ \\
\hline T. rugulosa F.Stein var. rugulosa & 5 & & & & & \\
\hline T. spinosa A.C.Stokes & & & & & & $\mathrm{X}$ \\
\hline T. sculpta Balech var. sculpta & 5 & & & & & \\
\hline T. similis Stokes & 2 & & & & & \\
\hline T. superba Svirenko emend. Deflandre & $2 ; 5$ & & & $\mathrm{x}$ & $\mathrm{x}$ & \\
\hline T. volvocina (Ehrenberg) Ehrenberg var. volvocina & $1 ; 2 ; 3 ; 4 ; 5$ & $\mathrm{x}$ & & $\mathrm{X}$ & & \\
\hline T. volvocina Ehrenberg var. compressa Deflandre & 2 & & & & & \\
\hline T. volvocinopsis Svirenko var. volvocinopsis & 5 & $\mathrm{x}$ & $\mathrm{x}$ & $\mathrm{X}$ & $\mathrm{x}$ & $\mathrm{x}$ \\
\hline Trachelomonas sp. 1 & & $\mathrm{x}$ & & & & \\
\hline
\end{tabular}

\section{CONCLUSÕES}

O estudo das 60 unidades amostrais coletadas nos municípios de São Sebastião do Passé, Candeias, Mata de São João, Pojuca e São Francisco do Conde, Região Metropolitana de Salvador, Bahia, Brasil, permitiu concluir que: 1. A área apresentou expressiva biodiversidade, tendo sido registrados 31 táxons; 2 . Três táxons constituem novos registros para a região Nordeste do Brasil (T. acanthophora Stokes, T. spinosa, $T$. lemmermannii var. acuminata); 3. Dois táxons constituem novos registros para o estado da Bahia: (T. hispida var. crenulatocollis, T. magdaleniana Deflandre); 4. Um táxon (Trachelomonas sp. 1) não pode ser encaixado em qualquer circunscrição de Trachelomonas já descrita e, muito provavelmente, é uma novidade para a ciência; 5 . 
Duas variedades são prováveis táxons novos para a ciência (T. kellogii var. 1, T. kellogii var. 2); 6. Dentre os táxons inventariados quatro foram exclusivos de São Sebastião do Passé; 7. Os municípios de Mata de São João, Pojuca e São Francisco do Conde apresentaram um táxon exclusivo cada, enquanto que o município de Candeias não apresentou nenhum; 8. O município de São Sebastião do Passé apresentou a maior riqueza de táxons (25) enquanto que o município de Candeias apresentou a menor riqueza (11); 9. T. volvocinopsis var. volvocinopsis foi o táxon mais representativo, considerando os cinco municípios amostrados, tendo sido registrado em 31 unidades amostrais;

\section{REFERÊNCIAS}

ALVES-DA-SILVA, S.M.; MENEZES, M. 2015. Euglenophyceae in Lista de Espécies da Flora do Brasil. Jardim Botânico do Rio de Janeiro. Disponivel em: <http://floradobrasil.jbrj.gov.br/jabot/floradobrasil/FB109138>

BICUDO, C.E.M.; MENEZES, M. 2006. Gêneros de algas de águas continentais do Brasil: chave para identificação e descrições. 2 ed. Rima, São Carlos.

BOURRELLY, P.C. 1970. Les algues d'eau douce: initiation à la systématique. Tome III. Les algues blues et rouges. Les Eugléniens, Peridiniens et Cryptomonadines. Paris: Éditions N. Boubée.

DUNLAP, J.R.; WALNE, P.L.; BENTLEY, J. 1983. Microarchitecture and elemental spatial segregation of envelopes of Trachelomonas lefevrei (Euglenophyceae). Protoplasma 117: 97-106.

FUENTES, E.V.; OLIVEIRA, H.S.B.; CORDEIRO-ARAÚJO, M.K.; SEVERI, W.; MOURA, A.N. 2010. Variação espacial e temporal do fitoplâncton do Rio de Contas, Bahia, Brasil. Revista Brasileira de Engenharia de Pesca 5(2): 13-25.

GUIRY in GUIRY, M.D.; GUIRY, G.M. 2016. AlgaeBase. World-wide electronic publication, National University of Ireland, Galway. http://www.algaebase.org. Acesso 3 Mai 2016.

LEEDALE, G.F. 1967. Euglenoid Flagellates. Englewood, New Jersey: Prentice Hall. LEEDALE, G.F. 1975. Envelope formation and structure in the euglenoid genus Trachelomonas. European Journal of Phycology 10: 17-41.

NORTON, T.A.; MELKONIAN, M.; ANDERSEN, R.A. 1996. Algal biodiversity. Phycologia 35: 308-326.

PARRA, O.O.; BICUDO, C.E.M. 1995. Introdução a la biología y sistemática de las algas continentales. Chile: Universidad de Concepción.

PEREIRA, A.J.S. 2016. O gênero Trachelomonas (Euglenophyceae) de duas áreas dos Marimbus (Baiano e Remanso), Chapada Diamantina, Bahia, Brasil. XX Seminário de Iniciação Científica da Universidade Estadual de Feira de Santana, Outubro de 2016, resumo expandido, p.1-4.

SANTANA, L.M. 2011. Análise física e química da água e estrutura da comunidade fitoplanctônica do rio Almada (sul da Bahia). Dissertação de Mestrado, Programa de PósGraduação em Sistemas Aquáticos Tropicais, Universidade Estadual de Santa Cruz, Bahia.

SEVERIANO, J.S.; MOURA, A.N.; BEZERRA-OLIVEIRA, H.S.; CORDEIROARAÚJO, M.K.; DANTAS, E.W. 2012. Microphytoplankton richness in Contas River, state of Bahia, northeastern Brazil. Checklist: Journal of Species Lists and Distribution 8(2): 218-223.

TELL, G.; CONFORTI, V. 1986. Euglenophyta Pigmentadas de la Argentina. Bibliotheca Phicologica 75: 1-301. 